\title{
Spinopelvic Sagittal Alignment in Patients With Adolescent Idiopathic Scoliosis
}

\author{
Seyed Hossein Vahid Tari ${ }^{1}$; Ebrahim Ameri Mahabadi ${ }^{2}$; Hasan Ghandehari ${ }^{2}$; Farshad \\ Nikouei $^{2, *}$; Ramin Javaheri ${ }^{2}$; Farshad Safdari ${ }^{3}$ \\ ${ }^{1}$ Orthopedic Spine Surgery Department, Rasoul-e-Akram Hospital, Iran University of Medical Sciences, Tehran, IR Iran \\ 2 Bone and Joint Reconstruction Research Center, Shafa Orthopedic Hospital, Iran University of Medical Sciences, Tehran, IR Iran \\ ${ }^{3}$ Bone Joint and Related Tissue Research Center, Shahid Beheshti University of Medical Sciences, Tehran, IR Iran \\ ${ }^{*}$ Corresponding author: Farshad Nikouei, Bone and Joint Reconstruction Research Center, Shafa Orthopedic Hospital, Iran University of Medical Sciences, Tehran, IR Iran. Tel \\ +98-9133160007, Fax +98- 2133542020, E-mail: farshadortho@gmail.com
}

Received: March 21, 2015; Revised: April 23, 2015; Accepted: June 11, 2015

Background: Recently, several authors have demonstrated the importance of sagittal spinopelvic alignment in patients with adolescent idiopathic scoliosis (AIS).

Objectives: In the current study, we investigated the preoperative spinal and pelvic sagittal parameters in the abovementioned patients. Patients and Methods: There were 50 consecutive patients with AIS (16 males and 34 females) in the current prospective study. In addition to the measurement of main scoliotic curve on posteroanterior X-rays, pelvic incidence(PI), pelvic tilt(PT), sacral slope(SS), lumbar lordosis (LL), and thoracic kyphosis (TK) were measured on lateral X-rays. Finally, the correlations between these parameters were investigated. We also compared the sagittal parameters between patients with main thoracic scoliosis (Lenke type I) and thoracolumbar/lumbar scoliosis (Lenke type V).

Results: Main scoliotic curve (MSC) was correlated with TK and PT. LL was correlated positively with PI and SS. Negative correlation was found between SS and PI. The sagittal parameters were the same in patients with Lenke type I and Lenke type V scoliosis. In addition, MSC and LL were significantly greater in patients with thoracolumbar/lumbar scoliosis, while TK was significantly greater in patients with main thoracic scoliosis. In the main thoracic group, MSC and TK were positively and PI was negatively correlated with PT. LL and SS had a positive correlation.

Conclusions: PI has a constant magnitude and is correlated with SS and LL. Postoperatively, pelvis can compensate for any change in LL. Therefore, the LL within fusion must be imposed in lordosis to the extent that the below fusion segments can compensate for intrafusion hypo/hyper lordosis to prevent the pelvis to compensate for hypo/hyper lordosis. This can result in a normal or near normal postoperative sagittal alignment. The authors recommend the surgeons to perform exact preoperative planning including the sagittal alignment of the pelvis in addition to spinal alignment.

Keywords: Adolescent Idiopathic Scoliosis; Sagittal; Alignment; Spinal Deformity

\section{Background}

Adolescent idiopathic scoliosis (AIS) is the most common type of idiopathic scoliosis and a challenging problem for spinal surgeons. Besides severe physical effects, its impressions on appearance and function of the patient, especially in females, is associated with several psychosocial consequences.

Spinal column is a sophisticated biomechanical construct, the normal function of which requires exact and ordered performance of its composing components in three planes. Based on this fact, although previously surgeons tried to regain the normal spinal alignment only in frontal plane, recently, authors have demonstrated that sagittal alignment is as important as frontal alignment (1-5). Sagittal spinal alignment significantly affects the quality of life and outcomes of spinal surgeries (1).

AIS represents a tridimensional deformity and regain- ing normal sagittal balance must be one of the goals of any surgical procedure (6). Previous studies have found some abnormalities in sagittal spinal alignment such as decreased thoracic kyphosis and hypo-lordosis in patients with AIS (3, 7-10). Vertebral rotation in thoracic curves forces the spinal column anterolaterally and vertebral migration in lumbar curves forces the spinal column posterolaterally (11). Considering the anatomical and biomechanical relationships among different parts of the body, it is expected that any spinal malalignment in sagittal plane cause some changes in other parts of the musculoskeletal system such as pelvis or lower extremities (12-18). Although in recent years some studies have investigated the sagittal alignment in patients with AIS, our knowledge is limited and more extensive studies are required for better understanding of the sagittal changes

Copyright (C) 2015, Iran University of Medical Sciences. This is an open-access article distributed under the terms of the Creative Commons Attribution-NonCommercial 4.0 International License (http://creativecommons.org/licenses/by-nc/4.0/) which permits copy and redistribute the material just in noncommercial usages, provided the original work is properly cited. 
of the spinal column and their effects on other parts of the body as well as their operative outcomes.

\section{Objectives}

In the current study, we aimed to measure the preoperative spinal and pelvic sagittal parameters in patients with AIS. In addition, we compared the spinopelvic sagittal parameters between patients with main thoracic scoliosis and the ones with thoracolumbar/lumbar scoliosis.

\section{Patients and Methods}

Between 2010 and 2011, there were 59 consecutive patients with AIS, admitted to Shafa Orthopedic Hospital, Tehran Iran. Patients with previous history of spinal or thoracic surgery, coronal curve smaller 20 degrees, hip or knee contracture, limb leg discrepancy, neuromuscular, metabolic or syndrome diseases and spondylolisthesis on X-rays were excluded. Furthermore, patients older than 18 years were excluded. Regarding these criteria, nine of the patients were excluded and the study was completed with 50 patients (16 males and 34 females) with a mean age of $16.8 \pm 4.3(11-35)$ years.

After exact clinical examinations, standard standing full-length postero-anterior (PA) and lateral and bending spinal X-rays were taken. For lateral standing X-rays, the shoulders were flexed to 90 degrees. On PAX-rays, the apical vertebrae were determined and the coronal curves were measured using the Cobb's method (Table 1). The main curve was determined as the most rigid curve with the greatest rotation of apical vertebra and curve angle (19). We used Lenke's classification system to determine the types of deformities.

To evaluate the sagittal spinal alignment, we measured the thoracic kyphosis (TK) and Lumbar Lordosis (LL) as explained in Table 1 . The spinopelvic alignment was investigated with measuring the sacral slope (SS), pelvic incidence (PI) and pelvic tilt (PT) (8). The methods of measurement of these parameters are presented in Table 1 . In the current study, the midway between the centers of two femoral heads was considered as the hip axis. All of the measurements were performed by two fellowships of spinal surgery. Before the study, the intra-and inter-observer reliability were measured $0.9 \mathrm{~m}$ in a pilot study for both of the surgeons. We compared the radiographic variables between patients with main thoracic curves (Lenke type I) and those with thoracolumbar/lumbar curves (Lenke type V).

Statistical analysis was performed with SPSS statistical software (version 15.0; SPSS, Chicago, IL). The correlations between different parameters were investigated by Pearson's correlation coefficient (r). For comparing the radiographic variables between different Lenke groups, independent samples T-test was used. P value $<0.5$ was considered significant.

\section{Results}

The main scoliotic curve (MSC) was $48.5^{\circ} \pm 24.7^{\circ}$ (range: $\left.20^{\circ}-107^{\circ}\right)$. For all the patients, TK and LL were $46.6^{\circ} \pm 20.5^{\circ}$ (range: $6^{\circ}-85^{\circ}$ ) and $43.2^{\circ} \pm 12.7^{\circ}$ (range: $16^{\circ}-68^{\circ}$ ), respectively. The means of PI, SS and PT were measured as $43.2^{\circ}$ $\pm 11.5^{\circ}\left(\right.$ range: $\left.6^{\circ}-62^{\circ}\right), 35.2^{\circ} \pm 7.4^{\circ}\left(\right.$ range: $\left.20^{\circ}-56^{\circ}\right)$ and $12^{\circ} \pm$ $11.9^{\circ}$ (range: $-8^{\circ}-52^{\circ}$ ), respectively.

We found that the size of MSC was correlated with TK and PT (Table 2). In addition, TK was correlated with PT (Table 2). LL was also correlated positively with PI and SS (Table 2). Negative and positive correlations were found in SS with PT and PI, respectively (Table 2). There was no other significant correlation between the measured variables. MSC and LL were significantly greater in patients with thoracolumbar/lumbar scoliosis. Conversely, TK was significantly greater in patients with main thoracic scoliosis (Table 3). In patients with AIS type V, we found no significant correlation between the sagittal parameters; however, in the main thoracic group, there were some correlations which are presented in Table 4. MSC and TK were positively and PI was negatively correlated with PT. LL and SS had a positive correlation.

\section{Table 1. Methods of Measuring the Spinopelvic Sagittal Parameters ${ }^{\text {a }}$ \\ Main scoliotic curve The angle between the intersecting perpendicular lines from the superior surface of the superior end vertebra and the inferior surface of the inferior end vertebra \\ Thoracic kyphosis The angle between the intersecting perpendicular lines from $\mathrm{T} 1$ and from the inflected vertebra transited from kyphosis to lordosis \\ Lumbar lordosis The angle between the intersecting perpendicular lines from the inflected vertebra from kyphosis to lordosis and the superior end plate of L5 \\ Pelvic incidence The angle between the perpendicular section of superior S1 end plate and the line joining the middle of the sacral plate and the hip axis

Sacral slope
The angle between the $S 1$ superior end plate and the horizontal line
The angle between the vertical line and the line joining the middle of the superior end plate of S1 and the
hip axis

\footnotetext{
a Abbreviations: L5, fifth lumbar vertebra; S1, first sacral vertebra; T1, first thoracic vertebra.
} 
Table 2. Statistically Significant Correlations Between Spinopelvic Sagittal Parameters in Patients With Adolescent Idiopathic Scoliosis (AIS) ${ }^{\text {a }}$

\begin{tabular}{lcc}
\hline Variables & Pearson's Correlation Coefficient & PValue \\
\hline MSC-TK & 0.373 & 0.008 \\
MSC-PT & 0.399 & 0.005 \\
TK-PT & 0.36 & 0.011 \\
LL-PI & 0.317 & 0.03 \\
LL-SS & 0.377 & 0.008 \\
PI-SS & 0.478 & 0.001 \\
SS-PT & -0.336 & 0.018 \\
\hline
\end{tabular}

a Abbreviations: MSC, main scoliotic curve; LL, lumbar lordosis; PI, pelvic inclination; PT, pelvic tilt; TK, thoracic kyphosis SS, sacral slope.

Table 3. Comparing the Demographic Characteristics and Sagittal Spinopelvic Parameters Between Patients With Lenke Type I and V Deformity ${ }^{\text {a }}$

\begin{tabular}{lccc}
\hline Variables & Main Thoracic Curve $(\mathbf{n}=\mathbf{1 8})$ & Thoracolumbar/Lumbar Curve $(\mathbf{n}=\mathbf{1 6})$ & P Value \\
\hline Age, $\mathbf{y}$ & $16.8 \pm 3.9$ & $16 \pm 1.8$ & NS \\
Gender & $4(22.2 \%)$ & & NS \\
\multicolumn{1}{c}{ Male } & $14(77.8 \%)$ & $7(43.75 \%)$ & \\
\hline Female & $40.4 \pm 18.5$ & $9(56.25 \%)$ & $<0.001$ \\
\hline MSC, $^{\circ}$ & $49.4 \pm 18.3$ & $48.6 \pm 22.3$ & $<0.001$ \\
TK, $^{\circ}$ & $39.1 \pm 8.8$ & $36.8 \pm 13.1$ & $<0.001$ \\
LL, $^{\circ}$ & $42.3 \pm 13.6$ & $52.8 \pm 7.1$ & NS \\
PI, $^{\circ}$ & $35.5 \pm 5.8$ & $44.4 \pm 6.4$ & NS \\
\hline SS, & & $38.6 \pm 8.7$ & NS \\
\hline PT,
\end{tabular}

${ }^{\mathrm{a}}$ MSC, main scoliotic curve; TK, thoracic kyphosis; PT, pelvic tilt; LL, lumbar lordosis; PI, pelvic inclination; SS, sacral slope; NS, not significant.

Table 4. Statistically Significant Correlation Between Spinopelvic Sagittal Parameters in Patients With Main Thoracic Adolescent Idiopathic Scoliosis (AIS) ${ }^{\text {a }}$

\begin{tabular}{lcc}
\hline Variables & Pearson's Correlation Coefficient & PValue \\
\hline MSC-PT & 0.586 & 0.011 \\
TK-PT & 0.513 & 0.029 \\
PI-PT & -0.582 & 0.011 \\
LL-SS & 0.656 & 0.006 \\
\hline
\end{tabular}

a Abbreviations: MSC, main scoliotic curve; LL, lumbar lordosis; TK, thoracic kyphosis; PI, pelvic inclination; PT, pelvic tilt; SS, sacral slope.

\section{Discussion}

Previously, it was demonstrated in healthy populations that spinal sagittal curves (TK and LL) and pelvic sagittal alignment (SS and PI) were correlated and any change in these segments was compensated by changes in other regions of the musculoskeletal system (12-18). During et al. showed that lumbothoracic spine was affected by the shape and orientation of the pelvis (PI) (20). PI is a constant morphological parameter which does not change after growth and in association with changes of posture or position of the pelvis. In addition, PI does not get affected by the three dimensional orientations of the pel- vis $(8,21)$. These characteristics made PI a useful parameter in assessing the sagittal pelvis alignment and based on the structural and biomechanical spinopelvic correlation, evaluating the spinal diseases.

The importance of sagittal balance encouraged surgeons to consider it in evaluation of several pathologic spinal conditions such as low back pain, disk herniation, degeneration, and scoliosis (1-5, 7-10, 15, 21-23). The three plane deformity concepts of scoliosis in recent years caused the researchers and surgeons to consider the alignment of the spinal column in sagittal and transverse 
planes in addition to the coronal plane only. It seems that understanding the common compensatory changes is an important and useful tool, which helps to improve the outcomes of treatments in these patients (15).

Jackson et al. evaluated the compensatory spinopelvic balance over the hip axis in normal subjects and patients with degenerative lumbar disk disease, spondylolisthesis and scoliosis and found clinical compensation for the balance occurred in all of the participants. However, compensation resulted in different characteristics and angular alignments in the groups. Even small differences in lumbar lordosis led to substantial changes in the spinopelvic alignment (15). In addition to the effects of race on spinopelvic alignment, Lonner et al. found that larger PI resulted in compensatory larger LL to maintain a neutral sagittal balance (16). Qiu et al. found that in patients with AIS, lumbosacral alignment affects the thoracic kyphosis which should be evaluated before surgical management (5). Some authors have demonstrated that LL has been correlated with SS and PI in patients with spinal disorders such as AIS (9, 24-26).

The findings of the current study confirmed the results of previous studies. We evaluated the correlations between different sagittal spinopelvic parameters and found that TK, PT and MSC were positively correlated. In addition, LL was positively correlated with PI and SS. One should know that each ambulatory patient requires to maintain a horizontal gaze while minimizing the energy expenditure (12). The spinopelvic alignment and balance in three planes is an important factor affecting the energy expenditure for standing and walking in healthy individuals and patients with different diseases (16). As previously mentioned, minimizing the energy expenditure in balanced walking and standing in patients with spinal deformity including sagittal malalignment necessitates significant compensatory mechanisms. Our findings, like previous studies, showed that PI, SS and LL were correlated. In other words, PI has a constant magnitude and controls SS directly and LL indirectly, which is of paramount importance. Pelvis determines the amount of LL through PI and may compensate for changing LL by anteverting, retroverting and changing PT and SS (26). Inattention to these compensatory mechanisms may result in subsequent postoperative sagittal malalignment (12).

Generally, postoperatively SS seems to be correlated with the LL distal to the fusion. Furthermore, LL distal to fusion is negatively correlated with LL within fusion. The magnitude of LL within fusion can be determined by the surgeon intraoperatively. Hence, the surgeon must determine LL within fusion in a manner that postoperative overall LL be congruent with the pelvic geometry. The within-fusion lumbar spine should be imposed in lordosis to the extent that prevents the compensatory mechanisms to reciprocate the postoperative hyper/hypolordosis through changing the pelvic position (26). Although we did not perform a study to investigate these compensatory changes in pelvis after scoliosis surgery, it was observed in our clinical experience. Wasylenko et al. demonstrated that when LL within fusion imposed by the Harrington system was insufficient, the LL below fusion increased substantially (27).

In the current study, we investigated whether sagittal spinopelvic parameters differ between patients with thoracic and lumbar AIS. Although the sagittal pelvic parameters were the same in the two groups, MSC and LL were significantly greater in lumbar scoliosis, whereas TK was significantly greater in thoracic scoliosis. In fact, the magnitude of TK and LL was influenced by the location of the curve, which demonstrated the tridimensional nature of the AIS. We also found some significant correlations between the measured parameters in patients with main thoracic deformity, while there was no statistically significant correlation in the Lenke type $\mathrm{V}$ group. In patients with main thoracic scoliosis, PI was correlated with PT, which was correlated significantly with MSC and TK. It may be understood that PI indirectly affects the magnitude of MSC and TK through some compensatory mechanisms or vice versa. However, more studies are required to investigate these potential relationships, which, if present, can affect the postoperative postural outcomes. The authors think that if there were more patients in the thoracolumbar/lumbar scoliosis group, it would be possible to find some correlations between the sagittal parameters.

Charlebois et al. found that TK was affected by the thoracic scoliotic curve, resulting in hypokyphosis patients with Kings type I, II and III, whereas LL was affected by pelvic configuration. However, these authors explained that pelvic parameters did not differ between patients with different curve types and consequently could not induce the development of a specific type of deformity (3). Increased PI was found in patients with thoracic or thoracolumbar AIS compared to normal controls and cannot be the potential determinant of the curve type, but it is likely a risk factor for the AIS development. Furthermore, they explained that thoracic hypokyphosis versus increased lumbar lordosis and pelvic incidence in patients with thoracic deformity can support the theory of anterior overgrowth. The anterior overgrowth also may be the cause of lumbar scoliosis (10). In another similar study, TK was affected by spinal deformity, while LL depended on pelvic configuration. Like our study, the scoliotic curve type was not correlated with sagittal pelvic configuration. Although the authors found a greater PI than the normal subjects in patients with AIS, they were unable to relate the increased PI and the pathogenesis of AIS (8).

These findings clarified the important role of exact preoperative planning for patients with AIS. Although there are several studies regarding the sagittal alignment of patients with AIS, there are several unsolved problems which should be investigated in future studies.

Like any other study, there were some limitations in our study. The current study was limited by the sample size. We also did not evaluate the postoperative sagittal align- 
ment. The postoperative findings can help to understand the role of preoperative sagittal parameters in final outcomes.

As a conclusion, like normal subjects, in patients with AIS, PI was correlated with SS and LL. Pelvis can compensate for any change in LL after the operation. Since PI has a constant magnitude, LL within fusion must be imposed in lordosis to the extent that the below fusion segments can compensate for intrafusion hypo/hyper lordosis to prevent the pelvis to compensate for hypo/hyper lordosis. The authors recommend that surgeons perform exact preoperative planning, including the sagittal alignment of the pelvis, in addition to spinal alignment. Including the pelvis in preoperative planning results in normal or near normal postoperative sagittal alignment. However, more future extensive studies are warranted.

It seems that the magnitude of TK and LL is influenced by the location of the curve due to the tridimensional nature of AIS. Furthermore, it is possible that PI indirectly affects the magnitude of MSC and TK through some compensatory mechanisms in patients with main thoracic scoliosis.

\section{Authors' Contributions}

Study concept, design, drafting the manuscript, and critical revision of the manuscript: Farshad Nikouei, Ebrahim Ameri Mahabadi, Hasan Ghandehari, Seyed Hossein Vahid Tari, Ramin Javaheri. Analysis and interpretation of data: Ramin Javaheri; Farshad Safdari. Study supervision: Hasan Ghandehari, Farshad Nikouei.

\section{References}

1. Lenke LG. Sagittal balance. J Neurosurg Spine. 2014;20(5):512.

2. Boissiere L, Bourghli A, Vital JM, Gille O, Obeid I. The lumbar lordosis index: a new ratio to detect spinal malalignment with a therapeutic impact for sagittal balance correction decisions in adult scoliosis surgery. Eur Spine J. 2013;22(6):1339-45.

3. Charlebois M, Mac-Thiong JM, Huot MP, de Guise JA, Skalli W, Labelle $H$. Relation between the pelvis and the sagittal profile in adolescent idiopathic scoliosis: the influence of curve type. Stud Health Technol Inform. 2002;91:140-3.

4. Vidal C, Ilharreborde B, Azoulay R, Sebag G, Mazda K. Reliability of cervical lordosis and global sagittal spinal balance measurements in adolescent idiopathic scoliosis. Eur Spine J. 2013;22(6):1362-7.

5. Qiu Y, Yin G, Cao XB. [The influence of thoracic kyphosis on sagittal balance of the lumbosacral spine in thoracic idiopathic scoliosis patients]. Zhonghua Wai Ke Za Zhi. 2008;46(16):1237-40.

6. Schmidt C, Liljenqvist U, Lerner T, Schulte TL, Bullmann V. Sagittal balance of thoracic lordoscoliosis: anterior dual rod instrumentation versus posterior pedicle screw fixation. Eur Spine J. 2011;20(7):1118-26.

7. Schwab FJ, Smith VA, Biserni M, Gamez L, Farcy JP, Pagala M. Adult scoliosis: a quantitative radiographic and clinical analysis. Spine (Phila Pa 1976). 2002;27(4):387-92.

8. Mac-Thiong JM, Labelle H, Charlebois M, Huot MP, de Guise JA. Sagittal plane analysis of the spine and pelvis in adolescent idiopathic scoliosis according to the coronal curve type. Spine (Phila Pa 1976). 2003;28(13):1404-9.
9. Sucato DJ, Agrawal S, O'Brien MF, Lowe TG, Richards SB, Lenke L. Restoration of thoracic kyphosis after operative treatment of adolescent idiopathic scoliosis: a multicenter comparison of three surgical approaches. Spine (Phila Pa 1976). 2008;33(24):2630-6.

10. Upasani VV, Tis J, Bastrom T, Pawelek J, Marks M, Lonner B, et al Analysis of sagittal alignment in thoracic and thoracolumbar curves in adolescent idiopathic scoliosis: how do these two curve types differ? Spine (Phila Pa 1976). 2007;32(12):1355-9.

11. Charles YP, Bouchaib J, Walter A, Schuller S, Sauleau EA, Steib JP Sagittal balance correction of idiopathic scoliosis using the in situ contouring technique. Eur Spine J. 2012;21(10):1950-6.

12. Klineberg E, Schwab F, Smith JS, Gupta MC, Lafage V, Bess S. Sagittal spinal pelvic alignment. Neurosurg Clin N Am. 2013;24(2):15762.

13. Vaz G, Roussouly P, Berthonnaud E, Dimnet J. Sagittal morphology and equilibrium of pelvis and spine. Eur Spine J. 2002;11(1):807.

14. Mac-Thiong JM, Berthonnaud E, Dimar J2, Betz RR, Labelle H Sagittal alignment of the spine and pelvis during growth. Spine (Phila Pa 1976). 2004;29(15):1642-7.

15. Jackson RP, Peterson MD, McManus AC, Hales C. Compensatory spinopelvic balance over the hip axis and better reliability in measuring lordosis to the pelvic radius on standing lateral radiographs of adult volunteers and patients. Spine (Phila Pa 1976) 1998;23(16):1750-67.

16. Lonner BS, Auerbach JD, Sponseller P, Rajadhyaksha AD, Newton $\mathrm{PO}$. Variations in pelvic and other sagittal spinal parameters as a function of race in adolescent idiopathic scoliosis. Spine (Phila Pa 1976). 2010;35(10):E374-7.

17. Guigui P, Levassor N, Rillardon L, Wodecki P, Cardinne L. [Physiological value of pelvic and spinal parameters of sagital balance: analysis of 250 healthy volunteers]. Rev Chir Orthop Reparatrice Appar Mot. 2003;89(6):496-506.

18. Vialle R, Levassor N, Rillardon L, Templier A, Skalli W, Guigui P. Radiographic analysis of the sagittal alignment and balance of the spine in asymptomatic subjects. J Bone Joint Surg Am. 2005;87(2):260-7.

19. Benli IT, Akalin S, Kis M, Citak M, Aydin E, Duman E. Fronta and sagittal balance analysis of late onset idiopathic scoliosis treated with third generation instrumentation. Kobe J Med Sci. 2001;47(6):231-53.

20. During J, Goudfrooij H, Keessen W, Beeker TW, Crowe A. Toward standards for posture. Postural characteristics of the lower back system in normal and pathologic conditions. Spine (Phila $\mathrm{Pa}$ 1976). 1985;10(1):83-7.

21. Barrey C, Jund J, Noseda O, Roussouly P. Sagittal balance of the pelvis-spine complex and lumbar degenerative diseases. A comparative study about 85 cases. Eur Spine J. 2007;16(9):1459-67.

22. Johnson RD, Valore A, Villaminar A, Comisso M, Balsano M. Pelvic parameters of sagittal balance in extreme lateral interbody fusion for degenerative lumbar disc disease. J Clin Neurosci. 2013;20(4):576-81.

23. Rajnics P, Templier A, Skalli W, Lavaste F, Illes T. The importance of spinopelvic parameters in patients with lumbar disc lesions. Int Orthop. 2002;26(2):104-8.

24. Labelle H, Roussouly P, Berthonnaud E, Transfeldt E, O'Brien $\mathrm{M}$, Chopin D, et al. Spondylolisthesis, pelvic incidence, and spinopelvic balance: a correlation study. Spine (Phila Pa 1976). 2004;29(18):2049-54.

25. Legaye J, Duval-Beaupere G, Hecquet J, Marty C. Pelvic incidence: a fundamental pelvic parameter for three-dimensional regulation of spinal sagittal curves. Eur Spine J. 1998;7(2):99-103.

26. Tanguay F, Mac-Thiong JM, de Guise JA, Labelle H. Relation between the sagittal pelvic and lumbar spine geometries following surgical correction of adolescent idiopathic scoliosis. Eur Spine J. 2007;16(4):531-6.

27. Wasylenko M, Skinner SR, Perry J, Antonelli DJ. An analysis of posture and gait following spinal fusion with Harrington instrumentation. Spine (Phila Pa 1976). 1983;8(8):840-5 